\title{
Elucidation of the tumoritropic principle of hypericin
}

\author{
M Van de Putte', T Roskams ${ }^{2}$, JR Vandenheede ${ }^{3}$, P Agostinis ${ }^{3}$ and PAM de Witte*,I \\ 'Laboratorium voor Farmaceutische Biologie, Faculteit Farmaceutische Wetenschappen, KU Leuven, Van Evenstraat 4, B-3000 Leuven, Belgium; \\ ${ }^{2}$ Afdeling Histochemie en Cytochemie, Faculteit Geneeskunde, KU Leuven, Belgium; ${ }^{3}$ Afdeling Biochemie, Faculteit Geneeskunde, KU Leuven, Belgium
}

Hypericin is a potent agent in the photodynamic therapy of cancers. To better understand its tumoritropic behaviour, we evaluated the major determinants of the accumulation and dispersion of hypericin in subcutaneously growing mouse tumours. A rapid exponential decay in tumour accumulation of hypericin as a function of tumour weight was observed for each of the six tumour models investigated, and a similar relationship was found between tumour blood flow and tumour weight. Moreover, there was a close correlation between the higher hypericin uptake in RIF-I tumours compared to RI tumours and tumour vessel permeability. To define the role of lipoproteins in the transport of hypericin through the interstitial space, we performed a visual and quantitative analysis of the colocalisation of hypericin and $\mathrm{DiOC}_{18}$-labelled lipoproteins in microscopic fluorescent overlay images. A coupled dynamic behaviour was found early after injection (normalised fluorescence intensity differences were on the whole less than 10\%), while a shifted pattern in localisation of hypericin and $\mathrm{DiOC}_{18}$ was seen after $24 \mathrm{~h}$, suggesting that during its migration through the tumour mass, hypericin is released from the lipoprotein complex. In conclusion, we were able to show that the tumour accumulation of hypericin is critically determined by a combination of biological (blood flow, vessel permeability) and physicochemical elements (affinity for interstitial constituents).

British Journal of Cancer (2005) 92, |406-|4|3. doi:10.1038/sj.bjc.66025 I2 www.bjcancer.com

(C) 2005 Cancer Research UK

Keywords: photodynamic therapy; hypericin; tumoritropic behaviour; perfusion; permeability; lipoproteins

Photodynamic therapy (PDT) involves the local or systemic administration of a photosensitising drug that, upon light irradiation and in the presence of oxygen, results in tumour destruction (Dolmans et al, 2003). We have recently been focusing on hypericin, a natural compound isolated from Hypericum plants (Lavie et al, 1995a), as a potent photosensitiser with a high antitumoral PDT efficacy (Chen and de Witte, 2000; Chen et al, $2001,2002)$. In the course of our study, we found that the compound accumulated to a large extent in tumour tissues. For instance, after systemic administration (i.p. $5 \mathrm{mg} \mathrm{kg}^{-1}$ hypericin), a 16 -fold higher concentration of hypericin in tumour tissue $v s$ surrounding healthy tissue (skin, muscle) was found in a subcutaneous P388 lymphoma tumour model growing in DBA/2 mice (Chen and de Witte, 2000). A fast clearance of hypericin in the liver, spleen, kidney and plasma was observed within $6 \mathrm{~h}$, while the peak concentration of hypericin in the tumour (maximal $8.7 \%$ of the injected dose per gram tissue $\left(\% \mathrm{ID} \mathrm{g}^{-1}\right)$ ) occurred at $24-48 \mathrm{~h}$ after drug administration. To confirm these data, a study using $\mathrm{C} 3 \mathrm{H}$ mice bearing subcutaneous RIF-1 fibrosarcoma tumours was performed. The tumour drug concentration increased rapidly over the initial hours and peaked $\left(5.5 \% \mathrm{ID} \mathrm{g}^{-1}\right)$ approximately $6 \mathrm{~h}$ after i.v. administration (Chen et al, 2001).

The tumoritropic characteristics of hypericin, therefore, imply that some (radio)labelled derivatives of the compound could be applied in the field of clinical radiodiagnosis, radiotherapy $\left({ }^{131} \mathrm{I}-\right.$

*Correspondence: Dr PAM de Witte;

E-mail: peter.dewitte@pharm.kuleuven.ac.be

Revised 3 I January 2005; accepted II February 2005 labelled) and possibly also in magnetic resonance imaging (MRI). In order to better understand the mechanistic background, the present paper addresses basic aspects and principles of the accumulation of hypericin in malignant tissue. Previous results have shown that, compared to normal cells, isolated malignant cells intrinsically do not take up more hypericin (Kamuhabwa et al, 2000). Therefore, the tumoritropic behaviour of hypericin should be envisioned as the result of molecular interactions with specific in vivo environmental, vascular and tumour tissue properties. In this paper, the tumour tissue accumulation of hypericin was examined as a function of tumour weight and a correlation with tumour perfusion and tumour vessel permeability was explored. Furthermore, we also investigated the intratumoral distribution of hypericin and its association with lipoproteins, aimed at defining the role of lipoproteins as regards the transport through the interstitial space.

\section{MATERIALS AND METHODS}

\section{Animals and tumour system}

The following tumour models were used: (a) mouse RIF-1 (radiation-induced fibrosarcoma) cells (kindly provided by Dr F Stewart, The Netherlands Cancer Institute, The Netherlands) subcutaneously (s.c.) grafted in female $\mathrm{C} 3 \mathrm{H} / \mathrm{Km}$ mice, (b) mouse MH22A hepatoma cells (kindly provided by $\mathrm{Dr} \mathrm{Z}$ Luksiene, Institute of Materials Science and Applied Research, Lithuania) s.c. grafted in female athymic nude mice, (c) human CaCo-2 colon carcinoma cells (kindly provided by Dr P Augustijns (KU Leuven)) s.c. grafted in female athymic nude mice, (d) human A431 cervix carcinoma cells (obtained from American Type Culture Collection 
(ATCC)) s.c. grafted in female athymic nude mice, (e) AY27 TCC (transitional cell carcinoma) rat cells (originally developed by Drs S Selman and JA Hampton (Ohio Medical College)) s.c. grafted in female athymic nude mice and (f) R1 rhabdomyosarcoma rat cells (kindly provided by Dr W Landuyt (KU Leuven)) s.c. grafted in female athymic nude mice.

Tumour cells $\left(2 \times 10^{6}\right)$ were inoculated s.c. on the depilated lower dorsum of female mice (weight range 21-25g, purchased from Charles River Laboratories (France) or B\&K Grimston (England)). Tumours were grown to different surface diameters ranging from 2 to $9 \mathrm{~mm}$ and to thicknesses ranging between 2 and $5 \mathrm{~mm}$, as measured by a calliper. These dimensions covered tumour weights from ca 5 to $200 \mathrm{mg}$.

All aspects of the animal experiment and husbandry were carried out in compliance with national and European regulations and were approved by the Animal Care and Use Committee of KU Leuven.

Statistical analysis was performed using Prism 4.00, GraphPad Software, San Diego, USA.

\section{Hypericin accumulation in tumour tissue}

Tumour-bearing animals were killed $6 \mathrm{~h}$ after i.v. tail injection of hypericin $\left(5 \mathrm{mg} \mathrm{kg}^{-1}\right)$. Hypericin (synthesised from emodin anthraquinone according to Falk et al (1993)) was dissolved in a mixture of $25 \%$ dimethylsulphoxide (DMSO), $25 \%$ polyethylene glycol (PEG) 400 and water $\left(2 \mathrm{mg} \mathrm{ml}^{-1}\right)$ immediately before injection. Tumour tissues were harvested, weighed and frozen at $-20^{\circ} \mathrm{C}$ until determination of the hypericin content. Similar tissue samples were taken from control mice. Extraction and quantification of tissue hypericin concentrations were performed as previously described (Chen et al, 2001).

\section{Tumour blood perfusion}

The RIF-1 and R1 tumour-bearing animals were used to quantify the tumour perfusion by means of spectrofluorometric determination of tumour FITC-dextran uptake (fluorescein isothiocyanate dextran, $M_{\mathrm{r}} 2 \times 10^{6}$, obtained from Sigma, St Louis, MO, USA), as described (Chen et al, 2002).

\section{Tumour vessel permeability}

The RIF-1 and R1 tumour-bearing animals were used to assess the tumour vessel permeability by a modification of the procedure described by Graff et al (2001). Evans blue dye (Sigma, St Louis, MO, USA) was dissolved in PBS $\left(5 \mathrm{mg} \mathrm{ml}^{-1}\right)$ and injected i.p. in tumour-bearing mice $\left(25 \mathrm{mg} \mathrm{kg}^{-1}\right)$. After $48 \mathrm{~h}$, the animals were killed and the dissected and weighed tumour tissues dissolved in $1 \mathrm{ml}$ of tissue solubiliser (Soluene 350; Packard Industries, Downers Grove, IL, USA) at $37^{\circ} \mathrm{C}$ overnight. The solution was allowed to cool before the addition of $2 \mathrm{ml}$ of ethyl acetate (Fisher Scientific, UK) and $2 \mathrm{ml}$ of $1 \mathrm{~N} \mathrm{HCl}$. Absorption of the upper phase was read at $626 \mathrm{~nm}$ using a UV/Visible Spectrophotometer (Ultrospec 2000 Pharmacia Biotech, Amersham Biosciences, Uppsala Sweden). Concentrations were determined from a standard curve of Evans blue dye.

\section{Intratumoral localisation of hypericin and $\mathrm{DiOC}_{18}$}

The intratumoral localisation of hypericin and $\mathrm{DiOC}_{18}\left(3,3^{\prime}\right.$ dioctadecyloxacarbocyanine, Molecular Probes Inc., Eugene, OR, USA) was investigated by i.v. administering hypericin $\left(5 \mathrm{mg} \mathrm{kg}^{-1}\right)$, immediately followed by $\operatorname{DiOC}_{18}\left(5 \mathrm{mg} \mathrm{kg}^{-1}\right)$ to RIF-1 tumourbearing animals (tumour weight range $150-200 \mathrm{mg}$ ) $5 \mathrm{~min}, 2 \mathrm{~h}, 6 \mathrm{~h}$ or $24 \mathrm{~h}$ before being killed. Prior to injection, $\mathrm{DiOC}_{18}$ was suspended $\left(2 \mathrm{mg} \mathrm{ml}^{-\mathrm{P}}\right)$ in $40 \%$ propylene glycol, $10 \%$ ethanol, $4 \%$ Tween 80 and water, after which the mixture was sonicated at $60^{\circ} \mathrm{C}$ for $2 \mathrm{~h}$. In another set of experiments, FITC-dextran (300 $\mathrm{mg} \mathrm{kg}^{-1}$, in PBS $30 \mathrm{mg} \mathrm{ml}^{-1}$ ) was administered $2 \mathrm{~min}$ before killing the RIF-1 tumour-bearing animals that had received hypericin $\left(5 \mathrm{mg} \mathrm{kg}^{-1}\right)$.

Tumour samples were immediately mounted in medium (Tissue Tek embedding medium, Miles Inc., Elkhart, IN 46515, USA) and immersed in liquid nitrogen. Different serial cryostat sections ( $5 \mu \mathrm{M}$ slices) were taken from each tumour. The first of two serial sections was stained with hematoxylin and eosin (H\&E) and the second was examined by fluorescence microscopy (Axioskop 2 plus equipped with a light-sensitive charge-coupled device digital camera (Carl Zeiss, Göttingen, Germany)). To specifically visualise hypericin, the Zeiss filter set 14 (ex: BP $510-560 \mathrm{~nm}$, em: LP $590 \mathrm{~nm}$ ) was used, whereas the distribution of $\mathrm{DiOC}_{18}$ or FITCdextran was examined with Zeiss filter set 10 (ex: BP $450-490 \mathrm{~nm}$, em: BP 515-565 nm).

Overlay fluorescence images were quantitatively analysed using a KS imaging software system (Carl Zeiss, Göttingen, Germany) by subdividing the images in 2269 square fields of $38.7 \mu \mathrm{m}^{2}$ (24 pixels/ field) and by measuring the average fluorescence intensity per field for hypericin and $\mathrm{DiOC}_{18}$, respectively. The data were normalised for the maximal fluorescence intensity of each compound, and expressed as percentage fluorescence intensity ( $\%$ f.i.). From these field-by-field data, scattergrams were constructed with axes representing the $\%$ f.i. of each compound. In addition, the absolute field-by-field differences in fluorescence intensity for

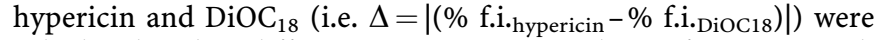
calculated. The differences were grouped in fractions with increments of $10 \%$ and the percentage of fields corresponding to each of the fractions was determined. In total, 15 overlay fluorescence images randomly taken throughout different tumours $(n=3)$ were analysed for each time interval.

\section{RESULTS}

\section{Tumour accumulation of hypericin}

After systemic administration of hypericin, its uptake in tumour tissue was studied as a function of tumour weight in six tumour models. As previous results using the RIF-1 tumour model had demonstrated, a peak concentration of hypericin in tumour tissue between 4 and $8 \mathrm{~h}$ after intravenous injection, a $6 \mathrm{~h}$ interval between administration and analysis was used in all cases. From Figure 1 it can be seen that, hinging on the tumour size, large intratumoral differences in hypericin accumulation exist. Typically, small tumours tended to accumulate three to four times more of hypericin relative to their weight, as compared to larger tumours (ranging from 50 to $200 \mathrm{mg}$ ). As a matter of fact, for each tumour model investigated, a rapid exponential decay in hypericin accumulation was observed from the smallest tumours followed by a plateau phase, on the average starting from a tumour weight of $50 \mathrm{mg}$. Of interest, when comparing intertumoral dissimilarities, a difference in overall tumour accumulation was found between the mouse RIF-1 fibrosarcoma and rat R1 rhabdomyosarcoma tumours (see Figure 2).

\section{Tumour blood perfusion and vessel permeability}

To further evaluate the intratumoral and intertumoral differences in hypericin accumulation disclosed in R1 and RIF-1 tumours, we analysed and compared their relative perfusion as well as their vessel permeability as a function of tumour weight. To measure tumour perfusion, FITC-dextran was i.v. injected 2 min before the animal was killed. At this short interval, extravasation of the FITCdextran complex is negligible (Shockley et al, 1992) and therefore the fluorescent tracer is entirely confined to the lumen of the (tumour) blood vessels. The amount of dye extracted from the 

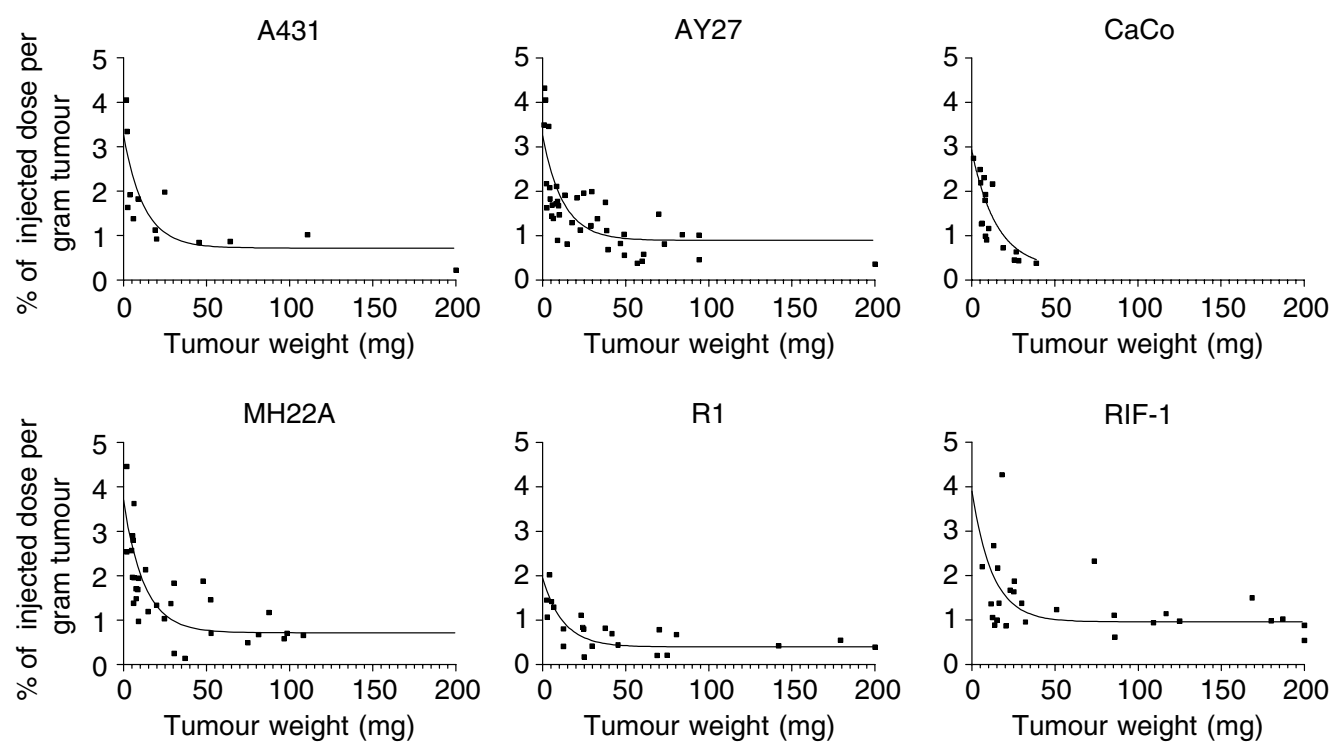

Figure I Accumulation of hypericin, expressed as \% of injected dose per gram of tumour, as a function of tumour weight. Animals bearing A43I, AY27, CaCo-2, MH22A, RI or RIF-I tumours were killed at $6 \mathrm{~h}$ after i.v. injection (tail) of hypericin $\left(5 \mathrm{mg} \mathrm{kg}^{-1}\right)$. Data points were fitted using one-phase exponential decay.

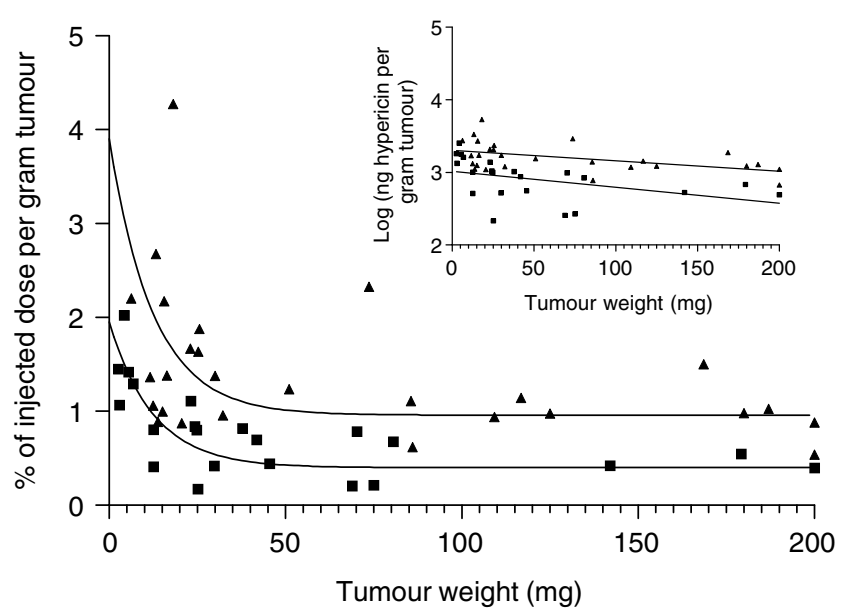

Figure 2 Comparison of the relative accumulation of hypericin in RI ( $)$ and RIF-I tumours $(\mathbf{\Delta})$. The accumulation of hypericin, expressed as $\%$ of injected dose per gram of tumour is shown as a function of tumour weight. Data points were fitted using one-phase exponential decay. The logarithm of the amount of hypericin recovered per gram of tumour tissue as a function of tumour weight is depicted in the inset (for RIF-I: $y=-0.001436 x+3.305$; for RI: $y=-0.002194 x+3.015)$. The data were statistically compared after linearisation using the two-way ANCOVA test (analysis of covariance). The difference in relative accumulation of hypericin in RI and RIF-I tumours was extremely significant $(P$-value $<0.000 \mathrm{I})$.

tumour was expressed as \% of the injected dose per gram of tumour as a function of tumour weight (see Figure 3). Intratumoral differences similar to the one found in case of the hypericin accumulation can be seen for both tumour types, that is, a weightdependent exponential decay in tumour blood flow followed by a plateau phase from tumour weights of $50 \mathrm{mg}$ on. As there was no significant difference between RIF-1 and R1 tumours (see Figure 3), an intertumoral variability in perfusion could not be found.

Tumour vessel permeability was measured by i.p. injection of Evans blue dye, a dye with a strong affinity for serum albumin. Extravasation of this dye is based on vessel permeability, which makes it a good marker for permeability measurements (Graff

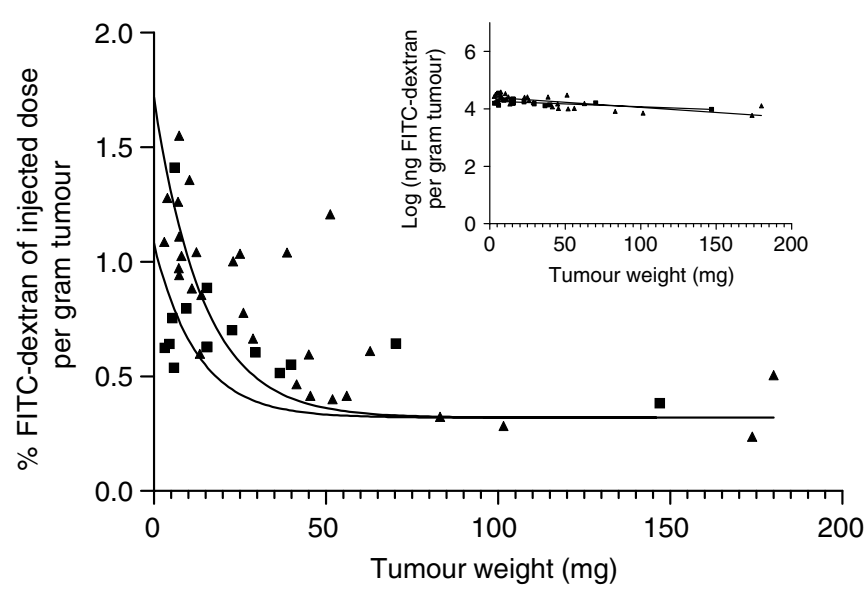

Figure 3 Perfusion of RIF-I ( $\mathbf{\Delta})$ and RI ( $)$ tumours. The amount FITC-dextran extracted is expressed as \% of injected dose per gram of tumour, as a function of tumour weight. FITC-dextran $\left(100 \mathrm{mg} \mathrm{kg}^{-1}\right)$ was i.v. injected 2 min before killing. Data points were fitted using one-phase exponential decay. The logarithm of the amount of FITC-dextran recovered per gram tumour tissue as a function of tumour weight is depicted in the inset (for RIF-I: $y=-0.003575 x+4.408$; for RI: $y=-0.001982 x+4.273)$. The data were statistically compared after linearisation using the two-way ANCOVA test (analysis of covariance). No significant difference between the perfusion of RIF-I and RI tumours was observed ( $P$-value: 0.064).

et al, 2001). Figure 4 shows the $\%$ of injected dose per gram of tumour $v s$ tumour weight, $48 \mathrm{~h}$ after administration. Except for small tumours $(<20 \mathrm{mg})$, the relative amount of Evans blue recovered from one tumour type was similar over a large range of tumour weight. However, major intertumoral differences between the permeability of vessels present in RIF-1 and R1 tumours were found.

\section{Intratumoral localisation of hypericin}

To track the intratumoral fate of hypericin, a fluorescence microscopy study was performed on sections of tumour biopsies 


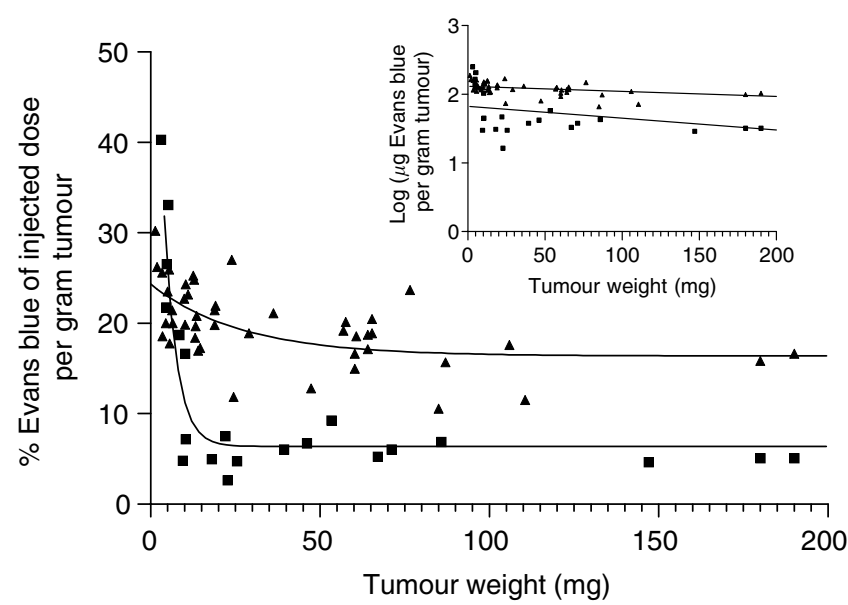

Figure 4 Extravasation of Evans blue in RIF-I tumours $(\mathbf{\Delta})$ and RI tumours ( $\mathbf{\square})$, expressed as \% of injected dose per gram of tumour, as a function of tumour weight. Data points were fitted using one-phase exponential decay. The logarithm of the amount of Evans blue recovered per gram tumour tissue as a function of tumour weight is depicted in the inset (for RIF-I: $y=-0.0007355 x+2.1$ I6; for $R I: y=-0.001724 x$ +1.826 ). The data were statistically compared after linearisation using the two-way ANCOVA test (analysis of covariance). The difference in vessel permeability in RI and RIF-I tumours was extremely significant ( $P$-value $<0.0001$ )

taken at different time points after systemic administration of the compound to RIF-1 tumour-bearing animals. Since blood-borne hypericin mainly associates with high-density lipoproteins (HDL) and other lipoproteins (Chen et al, 2001), it was of interest to verify whether the compound colocated intratumorally with the lipoproteins upon intravenous administration. For that purpose, $\mathrm{DiOC}_{18}$ was simultaneously injected with hypericin into the bloodstream. DiOC $_{18}$ is a green fluorescent analogue of $\mathrm{DiIC}_{18}$ (Pitas et al, 1981), a marker that by means of its very lipophilic $\mathrm{C}_{18}$ moieties avidly binds to lipoproteins without altering their affinity for the receptors.

Fluorescence microscopic analysis of tumour tissue, taken $5 \mathrm{~min}, 2,6$ and $24 \mathrm{~h}$ after administration of the compounds, revealed a shifting pattern, as a function of time, in the localisation of labelled lipoproteins and hypericin. At $5 \mathrm{~min}, \mathrm{DiOC}_{18}$ and hypericin were still confined to the luminal space of tumoral blood vessels (Figure 5A), as shown in separate experiments with FITCdextran that was injected 2 min before killing the animals (results not shown). Figure $5 \mathrm{~B}$ shows the situation after $2 \mathrm{~h}$, where red (hypericin) and green $\left(\mathrm{DiOC}_{18}\right)$ fluorescence are apparent in the vessels and in the perivascular region. Conversely, at $24 \mathrm{~h}$, a more homogeneous distribution is observed for hypericin in contrast to DiOC $_{18}$-labelled lipoproteins that show an irregular spreading (Figure 5C).

Quantitative measurements of colocalisation of hypericin and DiOC $_{18}$ were examined by a field-by-field analysis of fluorescent overlay images. Scattergrams revealed a good correlation between the percentage fluorescence intensity of hypericin and $\mathrm{DiOC}_{18^{-}}$ labelled lipoproteins at short intervals after coadministration of the compounds, whereas a poor correlation was observed at the later time points (Figure 6). For each time point, the percentage of fields that fall within fractions of grouped absolute differences $(\Delta)$ was scored (Figure 7, Table 1). A time-dependent shift in the distribution among the fractions can be seen, with an increased amount of larger differences between both compounds at longer time intervals. For instance, at $5 \mathrm{~min}$ and $2 \mathrm{~h}, 92 \pm 6.1$ and $88 \pm 5.6 \%$ (mean \pm s.d.), respectively, of the fields had $\Delta$ values less than $10 \%$, indicating that at these time points the distribution of fluorescence between hypericin and $\mathrm{DiOC}_{18}$ was similar.
However, $6 \mathrm{~h}$ after injection, significantly more fields displayed higher $\Delta$ values and at $24 \mathrm{~h}$ the majority of the fields exhibited $\Delta$ values of more than $10 \%$.

\section{DISCUSSION}

A basic understanding of the tumoritropic behaviour of hypericin would not only support the construction of hypericin derivatives with optimised PDT characteristics, but also sustain the development of labelled derivatives to extend the application of hypericins beyond the field of PDT. We therefore set out to gain a better insight in the major determinants of the accumulation and dispersion of hypericin in tumours.

Our study points out that, at least in the $5-50 \mathrm{mg}$ tumour weight range, the extent of hypericin uptake depends on the tumour weight, while in larger tumours the uptake remains constant. In the two models investigated (R1, RIF-1), an identical relationship was found between tumour blood flow and tumour weight. The fact that small tumours are relatively better perfused than larger ones has been documented before (Tozer et al, 1990; Hering et al, 1995). Since both the hypericin accumulation and the blood flow hinge to the same extent on the tumour weight, our data suggest that the hypericin accumulation in tumour tissue is critically dependent on the extent of local blood perfusion. Consistent with this hypothesis, our fluorescence microscopy analysis of RIF-1 tumour sections revealed a more homogeneous vessel distribution in small tumours, with a high percentage of functional vessels and a lack of necrotic areas. In contrast, large tumours have a more heterogeneous vessel distribution, with well-perfused regions in the periphery and less blood flow in the centrally located viable tumour regions. As a consequence, hypericin mainly accumulates in the periphery of these tumours (results not shown). Our results therefore support the concept that, especially in larger tumours, the intratumoral blood flow distribution is rather heterogeneous, both spatially and temporally (Jain, 2001).

Furthermore, we consistently found that the hypericin uptake in RIF-1 tumours was about twice as high as in R1 tumours. Since this difference cannot be accounted for by a dissimilar tumour perfusion, we investigated the permeability of the tumour vessels involved.

Extravasation of plasma constituents by means of convective currents across the microvascular wall originates from the incomplete or totally missing endothelial lining of the rapidly formed vessels in tumours growing beyond a mass of $10^{6}$ cells (Feng et al, 2000; Hashizume et al, 2000; Kuszyk et al, 2001). Since Evans blue accumulated more in RIF-1 than in R1 tumours over a large tumour weight range, it could be concluded that the larger amount of marker extravasated in RIF-1 tumours was due to an increased permeability of the tumour vessels. Hence, it is likely that the relatively low leakiness of the R1 tumour vessels explains the lower uptake of hypericin in R1 as compared to RIF-1 tumours. A similar correlation between tumoral photosensitiser uptake and tumour vessel permeability has been reported before (Roberts and Hasan, 1993).

Importantly, it was reported that hypericin binds to human lipoproteins at high molar ratios (up to 467 and 14 moles per $10^{4} \mathrm{Da}$ of low-density lipoproteins (LDL) and high-density lipoproteins (HDL), respectively) (Lavie et al, 1995b). In contrast to human plasma, mouse plasma contains a high HDL/LDL ratio (Kessel and Woodburn, 1993), so that mainly the hypericin-HDL complex is formed in the bloodstream of mice (results not shown). Once extravasated, the lipoproteins tagged with numerous hypericin molecules become embedded in the tumour interstitial fluid. At this stage, the hypericin-lipoprotein complexes can follow two routes leading to hypericin uptake and accumulation in tumour cells. Firstly, hypericin can comigrate with the lipoprotein microparticles throughout the interstitial space, followed by 

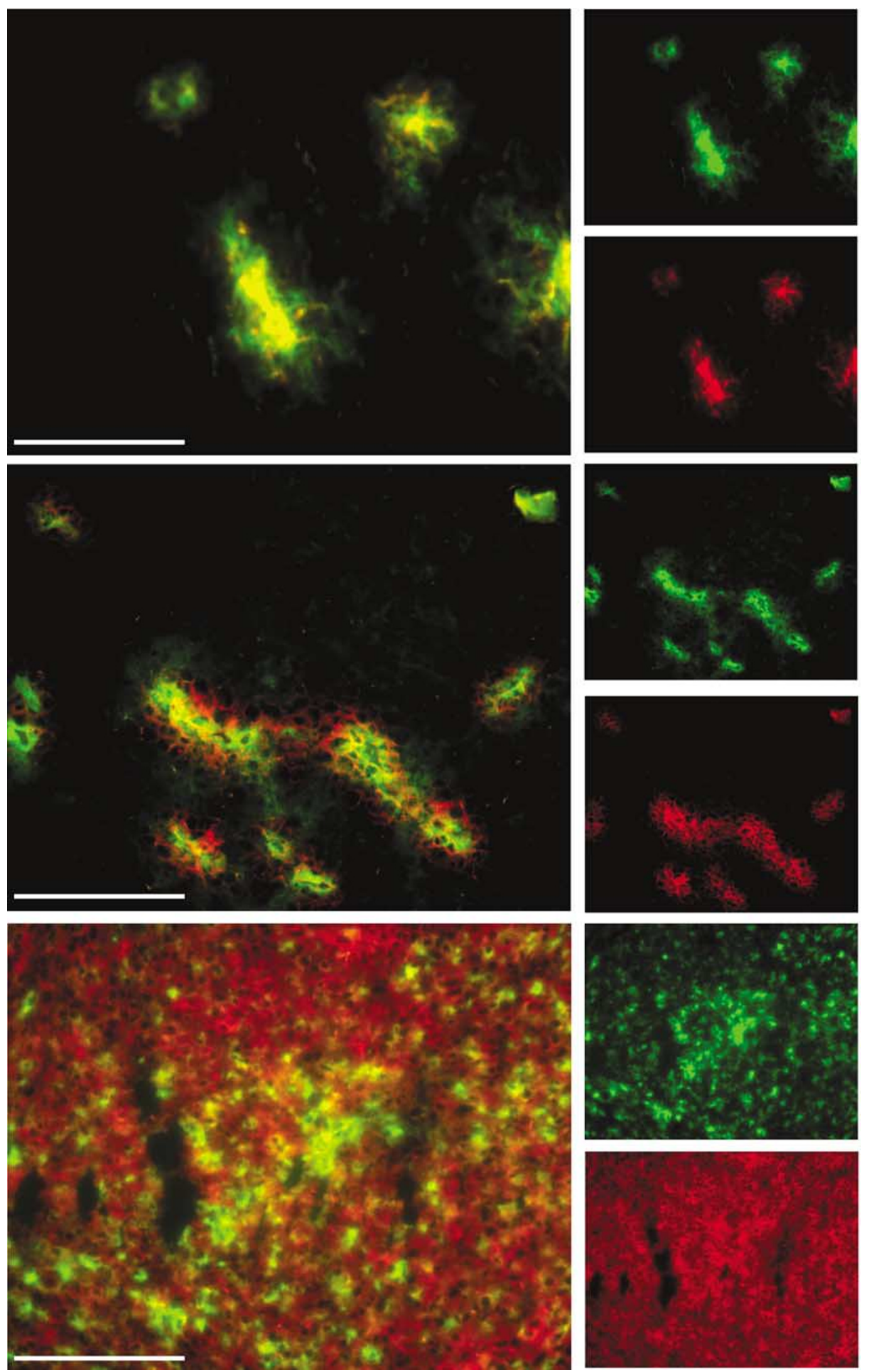

Figure 5 Fluorescence photomicrographs of $5 \mu \mathrm{m}$ RIF-I mouse tumour sections sampled at $5 \mathrm{~min}(\mathbf{A}), 2 \mathrm{~h}(\mathbf{B})$ and $24 \mathrm{~h}(\mathbf{C})$ after i.v. injection of $5 \mathrm{mg} \mathrm{kg}^{-1}$ hypericin (red fluorescence) and $5 \mathrm{mg} \mathrm{kg}^{-1} \mathrm{DiOC}_{18}$-labelled lipoproteins (green fluorescence). Overlay pictures show the combination of individual photomicrographs of hypericin in the left column, and of $\mathrm{DiOC}_{18}$ in the right column. Scale bar $=100 \mu \mathrm{M}$. 

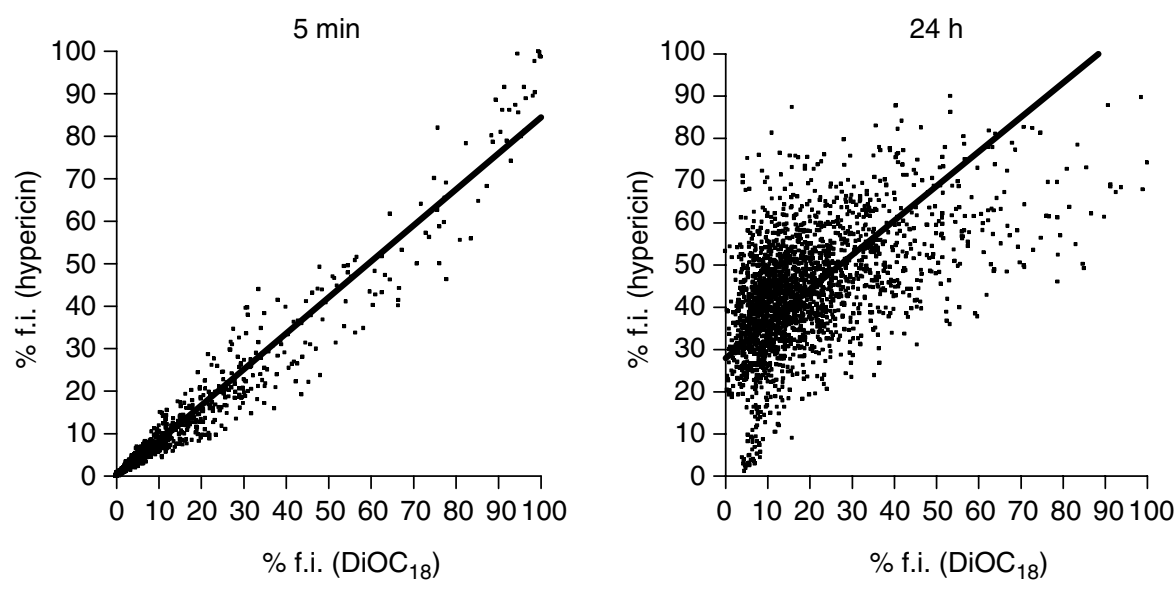

Figure 6 Scattergrams representing 2269 field-by-field relationships between normalised fluorescence intensities (\% fi.) of hypericin and DiOC 18 at 5 min $(r=0.99)$ and $24 \mathrm{~h}(r=0.52)$ after coadministration of the compounds. Results of representative fluorescent overlay images are shown.

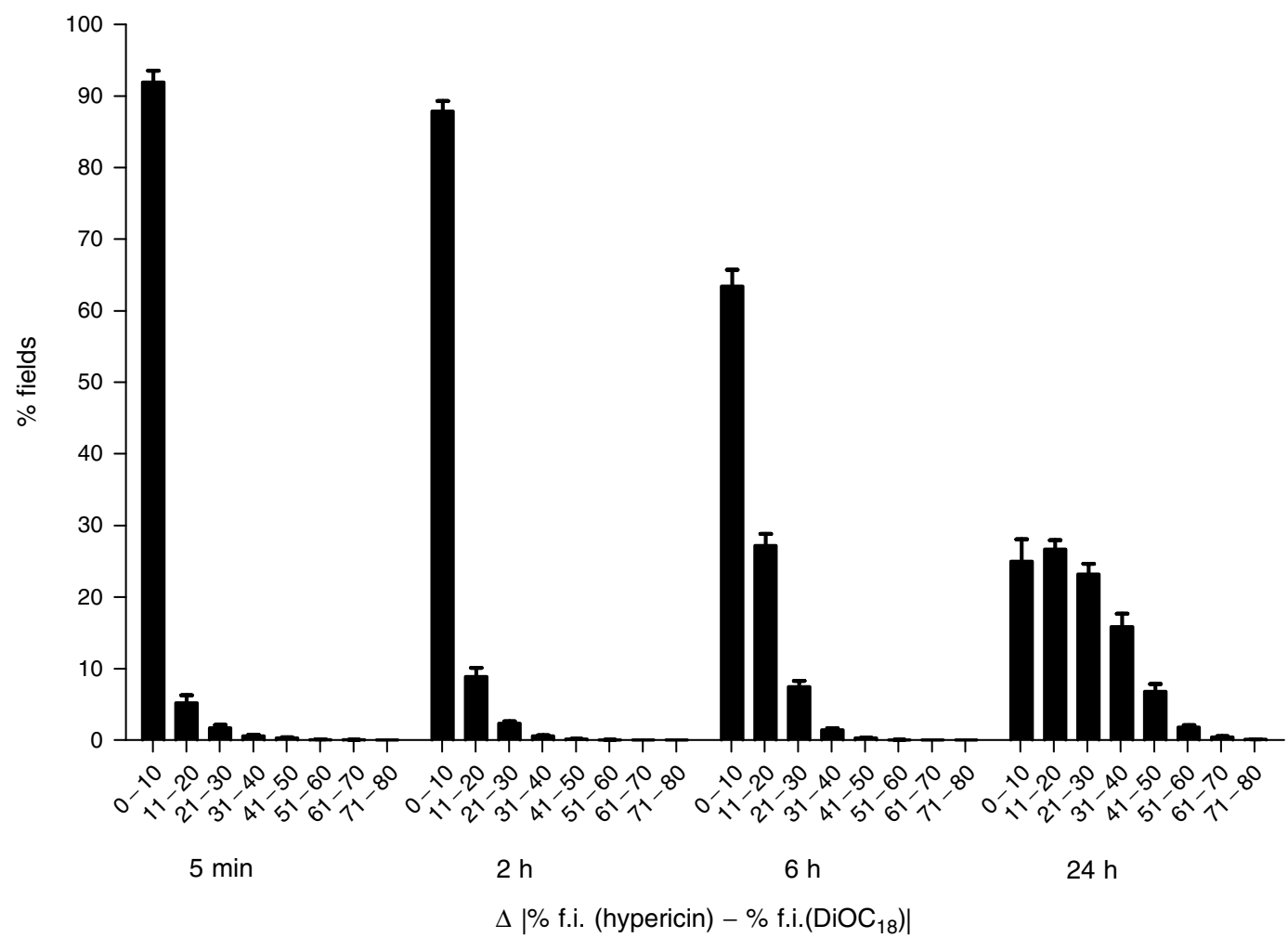

Figure 7 Time-dependent distribution of percentages of fields as a function of absolute differences $(\Delta)$ between normalised fluorescence intensities (\% f.i.) of hypericin and $\mathrm{DiOC}_{18} . \Delta$ Values were grouped in fractions with increments of $10 \%$. Data are expressed as mean \pm s.d. (average of the analysis of three tumours and 15 fluorescent overlay images per tumour). Relative field counts for $\Delta$ values between 80 and $100 \%$ were zero at all time points. Statistical analyses of the differences are shown in Table I.

receptor-mediated intracellular uptake. Of importance here, a correlation exists between the extent of association of some classes of photosensitisers with LDL and their efficiency of tumour targeting (Jori and Reddi, 1993). Alternatively, after collision of the microparticles with interstitial proteins or tumour cell membranes, hypericin can be released from the lipoprotein complex, followed by a lipoprotein-independent diffusion and intracellular uptake of the compound. A similar in vitro cellular uptake of hypericin in serum-free conditions has been documented (Siboni et al, 2002).

To investigate which route prevails, lipoproteins were marked with $\mathrm{DiOC}_{18}$, a compound that virtually irreversibly associates with lipoproteins. After simultaneous administration of hypericin and DiOC $_{18}$ to mice bearing RIF-1 tumours, fluomicroscopic analysis of tumour sections revealed that both compounds initially behaved similarly, indicating that hypericin and lipoproteins comigrate. However, later time intervals showed obvious differences in their intratumoral localisation. Thus, while the lipoproteins seem to be limited in their capacity to migrate through the interstitial space, hypericin spreads rather homogeneously over vascularised areas.

These results suggest that during its migration through the tumour mass, hypericin is released from the lipoprotein complex. In support of this notion, it is worth mentioning that unlike the 
Table I Statistical comparison of means of percentages of fields

\begin{tabular}{|c|c|c|c|c|}
\hline$\Delta(\%)$ & $5 \mathrm{~min}$ & $2 \mathrm{~h}$ & $6 \mathrm{~h}$ & $24 \mathrm{~h}$ \\
\hline $11-20$ & $6 \mathrm{~h} * * * 24 \mathrm{~h} * * * *$ & $6 h * * * * 24 h * * * *$ & $5 \min * * * 2 h * * *$ & $5 \mathrm{~min} * * * 2 \mathrm{~h} * * * *$ \\
\hline $41-50$ & $24 \mathrm{~h} * * *$ & $24 \mathrm{~h} * * *$ & $24 \mathrm{~h} * * * *$ & 5 min*** $2 h^{* * * *} 6 h^{* * * *}$ \\
\hline $51-60$ & NS & NS & NS & NS \\
\hline $61-70$ & NS & NS & NS & NS \\
\hline
\end{tabular}

Levels of significance are indicated as: $* P<0.05$, $* * P<0.01$, $* * * P<0.001$. For instance, $* * * 6 \mathrm{~h}$ (as mentioned in the 5 min column) means that the mean of $\%$ fields in the specific $\Delta$ fraction at $5 \mathrm{~min}$ is statistically different from the mean of \% fields in the same $\Delta$ fraction calculated at $6 \mathrm{~h}$, with $P<0.00 \mathrm{I}$. NS $=$ not significant. Statistical analysis was performed using the two-way ANOVA with Bonferroni test.

lipophilic $\mathrm{DiOC}_{18}$, hypericin is an amphiphilic compound that preferentially locates to the polar aprotic zone adjacent to the lipid - water interface (Lenci et al, 1995; Weitman et al, 2001). The phospholipid moiety in lipoproteins is situated at the surface of the microparticle, thereby allowing the associated hypericin molecules to dynamically interact with the immediate surrounding. This surrounding consists of phospholipid bilayers of plasma membranes of cancer cells and immune cells, and of proteins like collagen which are abundantly present in the interstitial space (Jain, 1987). Interestingly, it has been shown that hypericin associates with collagen resulting in a photodynamic effect, while another photosensitiser Chlorin $\mathrm{e}_{6}$ was ineffective (Yova et al, 2001).

In conclusion, the results of the present study indicate that the tumour accumulation of hypericin is critically determined by a combination of tumour-dependent and compound-dependent factors. As far as the tumour tissue is concerned, it is clear that an adequate vascularisation, blood flow and vessel permeability, all contribute to the ability of hypericin to accumulate in the tumour mass. On the other hand, once extravasated and locally delivered by a lipoprotein carrier, the compound itself can dramatically affect the level of its migration throughout the interstitial zone. Our study reveals that hypericin may have an affinity for some constituents typically present in the interstitium, and that there probably is a partitioning of the compound over lipoproteins, structural proteins and lipid bilayers of cancer and immune cells. This partitioning allows hypericin to spread rather homogeneously over the tumour mass.

The present study shows that hypericin accumulates less in tumoral sites with a poorly developed or afunctional vascular system, typical of larger tumours. Of importance, since the plasma clearance rate of hypericin is fast, following a two-phase exponential decay with half-lives of only 0.08 and $1.4 \mathrm{~h}$ (Chen et al, 2001), prolonging the exposure time of tumour tissue to blood-borne hypericin, that is by repeated i.v. injections or by intraperitoneal hypericin pumps, could result in an increased tumoral uptake and an improved homogeneous diffusion over the whole tumour mass. This is an interesting possibility, which is currently under investigation in our laboratory.

\section{ACKNOWLEDGEMENTS}

We thank Jan Maes (Laboratorium Farmaceutische Biologie) and Gerda Luyckx (Departement Pathologie) for excellent technical assistance. This work was supported by grants awarded by Fonds voor Wetenschappelijk Onderzoek-Vlaanderen (FWO Vlaanderen) and a Geconcerteerde Onderzoeksactie (GOA) of the Flemish Government.

\section{REFERENCES}

Chen B, de Witte P (2000) Photodynamic therapy efficacy and tissue distribution of hypericin in a mouse P388 lymphoma tumor model. Cancer Lett 150: 111-117

Chen B, Roskams T, Xu Y, Agostinis P, de Witte P (2002) Photodynamic therapy with hypericin induces vascular damage and apoptosis in the RIF-1 mouse tumor model. Int J Cancer 98: 284-290

Chen B, Xu Y, Roskams T, Delaey E, Agostinis P, Vandenheede JR, de Witte P (2001) Efficacy of antitumoral photodynamic therapy with hypericin: relationship between biodistribution and photodynamic effects in the RIF-1 mouse tumor model. Int J Cancer 93: 275-282

Dolmans DE, Fukumura D, Jain RK (2003) Photodynamic therapy for cancer. Nat Rev Cancer 3: 380-387

Falk H, Meyer J, Oberreiter M (1993) A convenient semisynthetic route to hypericin. Monatsh Chem 124: 339-341

Feng D, Nagy JA, Dvorak AM, Dvorak HF (2000) Different pathways of macromolecule extravasation from hyperpermeable tumor vessels. Microvasc Res 59: $24-37$

Graff BA, Bjornaes I, Rofstad EK (2001) Microvascular permeability of human melanoma xenografts to macromolecules: relationships to tumor volumetric growth rate, tumor angiogenesis, and VEGF expression. Microvasc Res 61: 187-198

Hashizume H, Baluk P, Morikawa S, McLean JW, Thurston G, Roberge S, Jain RK, McDonald DM (2000) Openings between defective endothelial cells explain tumor vessel leakiness. Am J Pathol 156: 1363-1380
Hering ER, Blekkenhorst GH, Jones DTL (1995) Tumor blood flow measurements using coincidence counting on patients treated with neutrons. Int J Radiat Oncol Biol Phys 32: 129-135

Jain RK (1987) Transport of molecules in the tumor interstitium: a review. Cancer Res 47: 3039-3051

Jain RK (2001) Delivery of molecular and cellular medicine to solid tumors. Adv Drug Deliv Rev 46: 149-168

Jori G, Reddi E (1993) The role of lipoproteins in the delivery of tumourtargeting photosensitizers. Int J Biochem 25: 1369-1375

Kamuhabwa AR, Agostinis P, D'Hallewin M-A, Kasran A, de Witte P (2000) Photodynamic activity of hypericin in human urinary bladder carcinoma cells. Anticancer Res 20: 2579-2584

Kessel D, Woodburn K (1993) Biodistribution of photosensitizing agents. Int J Biochem 25: 1377-1383

Kuszyk BS, Corl FM, Franano FN, Bluemke DA, Hofmann LV, Fortman BJ, Fishman EK (2001) Tumor transport physiology. Am J Roentgenol 177: $747-753$

Lavie G, Mazur Y, Lavie D, Meruelo D (1995a) The chemical and biological properties of hypericin - a compound with a broad spectrum of biological activities. Med Res Rev 15: 111-119

Lavie G, Mazur Y, Lavie D, Prince AM, Pascual D, Liebes L, Levin B, Meruelo D (1995b) Hypericin as an inactivator of infectious viruses in blood components. Transfusion 35: $392-$ 400 
Lenci F, Angelini N, Ghetti F, Sgarbossa A, Losi A, Vecli A, Viappiani C, Taroni P, Pifferi A, Cubeddu R (1995) Spectroscopic and photoacoustic studies of hypericin embedded in liposomes as a photoreceptor model. Photochem Photobiol 62: 199-204

Pitas RE, Innerarity TL, Weinstein JN, Mahley RW (1981) Acetoacetylated lipoproteins used to distinguish fibroblasts from macrophages in vitro by fluorescence microscopy. Arteriosclerosis 1: 177-185

Roberts WG, Hasan T (1993) Tumor-secreted vascular permeability factor/ vascular endothelial growth factor influences photosensitizer uptake. Cancer Res 53: 153 - 157

Shockley TR, Lin K, Nagy JA, Tompkins RG, Yarmush ML, Dvorak HF (1992) Spatial distribution of tumor-specific monoclonal antibodies in human melanoma xenografts. Cancer Res 52: 367-376
Siboni G, Weitman H, Freeman D, Mazur Y, Malik Z, Ehrenberg B (2002) The correlation between hydrophilicity of hypericins and helianthrone: internalization mechanisms, subcellular distribution and photodynamic action in colon carcinoma cells. Photochem Photobiol Sci 1: 483-491

Tozer GM, Lewis S, Michalowski A, Aber V (1990) The relationship between regional variations in blood flow and histology in a transplanted rat fibrosarcoma. Br J Cancer 61: 250-257

Weitman H, Roslaniec M, Frimer AA, Afri M, Freeman D, Mazur Y, Ehrenberg B (2001) Solvatochromic effects in the electronic absorption and nuclear magnetic resonance spectra of hypericin in organic solvents and in lipid bilayers. Photochem Photobiol 73: 110-118

Yova D, Hovhannisyan V, Theodossiou T (2001) Photochemical effects and hypericin photosensitized processes in collagen. J Biomed Opt 6: 52-57 